\title{
Population study of causes, treatment, and outcome of infertility
}

\author{
M G R HULL, C M A GLAZENER, N J KELLY, D I CONWAY, P A FOSTER, R A HINTON, \\ C COULSON, P A LAMBERT, E M WATT, K M DESAI
}

\begin{abstract}
Specialist infertility practice was studied in a group of 708 couples within a population of residents of a single health district in England. They represented an annual incidence of $1 \cdot 2$ couples for every 1000 of the population. At least one in six couples needed specialist help at some time in their lives because of an average of infertility of $2 \frac{1}{2}$ years, $71 \%$ of whom were trying for their first baby. Those attending gynaecology clinics made up $10 \%$ of new and $22 \%$ of all attendances. Failure of ovulation (amenorrhoea or oligomenorrhoea) occurred in $21 \%$ of cases and was successfully treated (two year conception rates of $96 \%$ and $78 \%)$. Tubal damage $(14 \%)$ had a poor outlook $(19 \%)$ despite surgery. Endometriosis accounted for infertility in $6 \%$, although seldom because of tubal damage, cervical mucus defects or dysfunction in $3 \%$, and coital failure in up to $6 \%$. Sperm defects or dysfunction were the commonest defined cause of infertility $(24 \%)$ and led to a poor chance of pregnancy $(0-27 \%)$ without donor insemination. Obstructive azoospermia or primary sper-
\end{abstract}

University of Bristol Department of Obstetrics and Gynaecology, Bristol Maternity Hospital, Bristol BS2 8EG

M G R HULL, MD, FRCOG, reader, consultant

C M A GLAZENER, MD, BMS, research registrar

N J KELLY, BSC, chief technician

D I CONWAY, MD, MRCOG, lecturer, senior registrar

PA FOSTER, MRCOG, MRACOG, lecturer, senior registrar

Fertility Clinic, Central Health Clinic, Bristol BS2 0JD

R A HINTON, MB, DRCOG, associate specialist

C COULSON, MB, DRCOG, clinical assistant

P A LAMBERT, MRCGP, DRCOG, clinical assistant

E M WATT, MB, DRCOG, clinical assistant

Department of Urology, Southmead Hospital, Bristol BS10 5NB

K M DESAI, FRCS, research registrar

Correspondence to: $\mathrm{Mr}$ Hull. matogenic failure was uncommon (2\%) and hormonal causes of male infertility rare. Infertility was unexplained in $28 \%$ and the chance of pregnancy (overall $72 \%$ ) was mainly determined by duration of infertility.

In vitro fertilisation could benefit $80 \%$ of cases of tubal damage and $25 \%$ of unexplained infertility-that is, $18 \%$ of all cases, representing up to 216 new cases each year per million of the total population.

\section{Introduction}

The 1984 Warnock report commented on the lack of statistics on infertility.' The common estimation is that $10 \%$ of the population are infertile. This takes no account of the duration of infertility, nor whether it is primary or secondary. Failure to conceive after a year is taken to be abnormal as $90 \%$ of fertile couples successfully conceive within that time. ${ }^{2}$ Permanent infertility might be rare, but that is irrelevant to infertile couples wanting a child, or to those who already have a child but are unable to conceive again. The usual distribution of causes of infertility is unknown because of the biased interests of the reporting clinics. We studied the incidence and range of causes of infertility in a representative British population, at least among those reaching specialist clinics; the estimated need for treatment; and its success. The findings should therefore be of general and administrative interest throughout Britain and similar countries. We focused on the residents of a particular health district and drew on information mainly from a single clinic attended by most of the infertile couples from that district.

\section{Patients and methods}

The Bristol and Weston District Health Authority manages a population of nearly 400000 living in the southern half of Bristol, the town of Westonsuper-Mare 20 miles away, and the Avon countryside between. Services for infertility are provided in various ways by general practitioners, 
gynaecologists, urological surgeons, endocrinologists, and others. The extent of the service by general practitioners is not known, but most infertile couples receiving specialist service attend a large gynaecological infertility clinic at the Central Health Clinic, Bristol, and a smaller complementary gynaecological endocrinology clinic at the Bristol Royal Infirmary, under the care of one gynaecologist (MGRH). Information from all other gynaecologists in this district showed that they undertook only $23 \%$ of the infertility work for local residents.

Another large gynaecological infertility clinic in the adjoining district provides a similar level of service along with gynaecology for the other residents of the Bristol area. The natural crossboundary flow of patients between the health districts of Bristol gave rise to a net inflow to the Bristol and Weston district amounting to $9 \%$ of its gynaecology patients resident in Avon at the time of our study. Presumably, this applied equally to patients attending the gynaecological and endocrinology infertility clinics. A further $13 \%$ of couples attending those clinics were referred from outside the health districts of Bristol and were therefore excluded from the study.

Most infertile men requiring the specific help of an endocrinologist or a urological surgeon first attended the gynaecological infertility clinics with their wives, but those referred directly were included in our study. Men and women wanting reversal of operative sterilisation were excluded.

The 708 couples studied formed three consecutive series: 515 attending the main gynaecological infertility clinic during part of 1982 and 1983, 172 attending the gynaecological endocrinology clinic during four years up to that time; and 21 referred directly to specialists in male infertility in 1982 and 1983. Infertility was defined as failure to achieve any pregnancy (including a miscarriage) for at least 12 months. Basic investigations have been described elsewhere, with particular emphasis on those for ovulatory failure and induction. ${ }^{4}$ In addition to a complete medical history and physical examination, they included: measurements of basal serum follicle stimulating hormone, prolactin, and thyroid hormone concentrations (in the woman); measurements of mid-luteal serum progesterone concentrations in two or three menstrual cycles ${ }^{5}$; seminal analysis ${ }^{6}$; postcoital testing of sperm penetration of cervical mucus ${ }^{7}$; and in vitro testing of sperm penetration of mucus in cases of negative postcoital tests. ${ }^{8}$ Laparoscopy to investigate pelvic disease and tubal state was reserved until simpler investigations and appropriate treatment for other defined disorders had been carried out for several months, unless the chlamydial antibody titre was high, suggesting the likelihood of inflammatory damage. ${ }^{9}$ Thus many conceived meanwhile, and barely half the women patients eventually underwent laparoscopy. Other investigations were carried out as necessary.

All couples were advised of their chance of conception, on proper coital timing, and how to recognise preovulatory secretion of cervical mucus to aid coital timing and postcoital testing. Treatments included all methods of ovulation induction, ${ }^{+}$artificial insemination, hormonal treatment for endometriosis, and pelvic or tubal surgery as required. Our study included artificial insemination (by the high intracervical and intrauterine method) using only the husband's semen to treat oligoasthenoteratozoospermia ("oligospermia") (overall motile normal sperm density $<4 \times 10^{6} / \mathrm{ml}^{8}$ and minimum crude sperm density $>1 \times 10^{6} / \mathrm{ml}$ ) and failure of sperm penetration of mucus. Results with donor insemination were excluded as it does not provide a true cure. In women with obvious appreciable pelvic or tubal disease tubal surgery was limited to those (about half) with complete occlusive disease, and to others with only unilateral or partial occlusion if infertility was prolonged in the absence of any other factor. Surgery was performed under magnification using loupes and the usual modern techniques of careful tissue handling with glass rods, constant irrigation, low power diathermy, complete haemostasis, and fine non-reactive suture materials. Women with minor endometriosis that was not causing damage were not treated because the importance of their condition for fertility is not clearly defined and hormonal treatment offers no proved benefit. ${ }^{10}$

The group with unexplained infertility included couples in which the menstrual cycle of the woman was normal (3-6 weeks in length); seminal analysis of the man yielded normal results ${ }^{6}$; the postcoital sperm penetration of mucus was normal'; there was normal coital activity at least twice a week; and laparoscopic findings were normal, unless pregnancy occurred beforehand, in which case the pelvic and tubal state was assumed to be normal. For practical purposes the group with unexplained infertility also included couples with minor abnormalities of uncertain importance, particularly oligospermia but normal sperm penetration of mucus, minor endometriosis without structural damage, and minor pelvic adhesions without obvious limitation of mobility or damage to essential structures, particularly the tubal fimbriae. All these couples received advice only and no specific treatment.

A diagnosis of luteal deficiency based on reduced progesterone concentrations in two or three cycles ${ }^{4}$ was not distinguished from unexplained infertility. Given normal menstrual cycles, it now seems to occur randomly, as in normal women, with no important contribution to protracted infertility. ${ }^{8}$

Results of treatment have been expressed as time specific, cumulative conception rates calculated using the life table method to allow for those couples who were not studied as long as others. ${ }^{3}$ The duration of observation was timed from the start of treatment or, in the case of unexplained infertility, from first attendance at the clinic. Statistical comparison between groups was made using the $t$ test for proportions. Similarly, the results were also compared with the highest rates reported in couples with proved fertility. ${ }^{23}$ Outcome of pregnancy was not included in this study as we have previously shown that pregnancy is not unusually complicated in most groups ${ }^{4}$ but about a quarter of the pregnancies were tubal in the group with tubal damage.

\section{Results}

After excluding couples referred from outside the Bristol area and allowing for the natural excess from adjoining health districts within this area and other couples seen by gynaecology colleagues the average number of couples resident in the Bristol and Weston health district attending specialist infertility clinics in 1982 and 1983 was 472 each year. Of these, 466 attended gynaecological infertility clinics at least initially. This figure, based on official audits that can be made available, represents $10 \%$ of all new gynaecology patients (taking each couple as a single case) resident in this district and $22 \%$ of all attendances of such patients at gynaecology clinics, patients with infertility attending on average about five times and patients with other gynaecological complaints twice.

The total district population was 393000 , of whom 204000 were girls and women, including 84100 aged 15-44. The lifetime incidence of infertility requiring specialist help could therefore be calculated, based on the average number of women in each year aged $15-44$ that is, $84100 / 30=2803$. (A narrower age range might have been used, or the total female population divided by the average life expectancy, but would have made little difference.) Thus the incidence of infertility (primary and secondary) in this district appeared to be $472 / 2803=0 \cdot 168(17 \%)$.

Of the $\mathbf{4 7 2}$ couples each year, $78 \%$ attended the particular clinics under study and therefore had accurate individual clinical information, taken to be representative of the whole group. Among the 708 couples studied the average duration of infertility was 29 months (range 1-13 years), the average age of the women was 28 years ( $18-46$ years), $59 \%$ being nulligravid, and the average age of the men was 31 years (18-64 years). The average age of the nulligravid women was 27.5 years and their duration of infertility 30 months; compared with the average age of primigravid women, who can be assumed to have started trying to conceive 12 months before on average, of 24.5 years at the time of delivery at Bristol Maternity Hospital

Table I gives the distribution of causes of infertility in the 708 couples studied as initially classified by the presenting features that were found, and corrected as above to the annual figure of 472 couples and related to parity. Investigations were incomplete in $9 \%$ of couples because they gave up or moved away from the district. The $41 \%$ of women overall who were parous (that is, had had any pregnancy) included $11 \%$ who had had a therapeutic termination of pregnancy and $29 \%$ who had had a child (or at least a pregnancy of 28 weeks or more)

The causes have been reorganised into a final, simpler, diagnostic classification, and the results are given in table II. The group with postcoital failure subclassified by in vitro testing of sperm penetration of mucus given in table I was redistributed to mucus, sperm, and presumptive coital dysfunction groups in table II. Oligospermia was excluded as an abnormality when associated with normal penetration of mucus, and azoospermia due to obstruction, primary spermatogenic failure, or hypogonadotrophism was separated from the group with sperm defects. There were parous women in every diagnostic group, and the proportion in each group was compared with the proportion in the remainder with the $\chi^{2}$ test. There was significantly higher incidence of both patients who had had a termination of pregnancy and children in the tubal group $(p<0.01)$, of patients who had had a termination in the groups with coital failure $(p<0.05)$, and of children in the group with unexplained infertility $(p<0.01)$. There was significantly reduced parity in the groups with endometriosis $(p<0.01)$, mucus defects $(p<0.01)$, and sperm defects $(p<0.01)$.

Figure 1 shows the results of treatment confined to 584 couples with a single cause of infertility or with unexplained infertility compared with normal. The group with amenorrhoea ( 95 cases) was treated by whatever method was appropriate as previously reported, excluding women with primary ovarian failure, ${ }^{4}$ and the conception rates were normal, reaching $96 \%$ after two years. In the group with oligomenorrhoea ( 89 cases) the rate reached $78 \%$, there being a significant shortfall compared with the normal group and the group with amenorrhoea $(p<0 \cdot 01)$, mainly due to unsuccessful treatment of polycystic ovary disease. ${ }^{11}$ In the group with unexplained infertility (196 cases) the rate reached $72 \%$, significantly less than normal $(p<0 \cdot 01)$. In the group with oligospermia ( 38 cases), which included failure of sperm penetration of mucus but excluded azoospermia, the rate was $11 \%$, and in the group with only failure of sperm penetration of mucus (31 cases) it

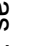

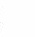


TABLE I-Annual causes of infertility as initially classified by presenting features and related to parity in new couples resident in the Bristol and Weston health district attending specialist clinics

\begin{tabular}{|c|c|c|c|c|c|}
\hline \multirow[b]{2}{*}{ Cause of infertility } & \multirow[b]{2}{*}{ No of couples with each cause } & \multirow{2}{*}{$\begin{array}{c}\% \text { Of couples } \\
(n=472)\end{array}$} & \multicolumn{3}{|c|}{ Previous pregnancy $(\%)$} \\
\hline & & & Any & Termination of pregnancy & Child* \\
\hline Ovulatory failure: & 97 & 21 & & & \\
\hline Amenorrhoea $†$ & 31 & 7 & 26 & 4 & 16 \\
\hline Oligomenorrhoea & 66 & 14 & 45 & 6 & 36 \\
\hline Tubal damage, adhesions $\ddagger$ & 68 & 14 & 70 & 20 & 44 \\
\hline Endometriosis: & 27 & 6 & & & \\
\hline With damage & 10 & 2 & 17 & 8 & 8 \\
\hline Without damage & 17 & 4 & 24 & 5 & 19 \\
\hline Primary mucus failure & 6 & 1 & 14 & 0 & 14 \\
\hline Failure of postcoital sperm penetration of mucus (semen and mucus of normal & & & & & \\
\hline appearance): & 61 & 13 & & & \\
\hline Mucus dysfunction, antibodies & 10 & 2 & 0 & 0 & 0 \\
\hline Sperm dysfunction, antibodies & 35 & 8 & 30 & 20 & 20 \\
\hline Normal in vitro: ? coital dysfunction & 16 & 3 & 56 & 11 & 33 \\
\hline Oligospermia (oligoasthenoteratozoospermia): & 71 & 15 & & & \\
\hline With failure of penetration of mucus & 57 & 12 & 22 & 4 & 15 \\
\hline With normal penetration of mucus & 14 & 3 & 44 & 6 & 33 \\
\hline Azoospermia and virtual azoospermia: & 28 & 6 & & & \\
\hline Primary spermatogenic failure & 19 & 4 & 36 & 14 & 21 \\
\hline Obstructive & 8 & 2 & 14 & 0 & 14 \\
\hline Hypogonadotrophic & 1 & & 0 & 0 & 0 \\
\hline Coital failure & 10 & 2 & 60 & 40 & 0 \\
\hline Other rare causes & 6 & 1 & & & \\
\hline Incompletely investigated & 44 & 9 & & & \\
\hline Unexplaineds & 133 & 28 & 47 & 10 & 41 \\
\hline Total No of causes $\|$ & 551 & & & & \\
\hline Total $\%$ of couples" & & & 41 & 11 & 29 \\
\hline
\end{tabular}

${ }^{\star}$ Child $=$ gestation at least 28 weeks, not necessarily successful.

†Includes three women with primary ovarian failure, which is untreatable to induce ovulation.

łIncludes three women with endometriosis.

§Defined by exclusion of all preceding causes, but includes some patients with minor abnormalities of doubtful importance: tubal adhesions ( 2 ), endometriosis (11), and oligospermia with normal postcoital penetration of mucus (7).

115\% Of couples have two or more causes of infertility.

TABLE II-A nnual causes of infertility by the final diagnostic classification and related to parity in new couples resident in the Bristol and Weston health district attending specialist clinics

\begin{tabular}{|c|c|c|c|c|c|}
\hline \multirow[b]{2}{*}{ Cause of infertility } & \multirow[b]{2}{*}{ No of couples with each cause } & \multirow{2}{*}{$\begin{array}{c}\begin{array}{c}\% \text { Of couples } \\
(n=472)\end{array} \\
\text { (n) }\end{array}$} & \multicolumn{3}{|c|}{ Previous pregnancy $(\%)$} \\
\hline & & & Any & Termination of pregnancy & Child $^{\star}$ \\
\hline Ovulatory failure $\nmid$ & 97 & 21 & 39 & 6 & 30 \\
\hline Tubal damage $\ddagger$ & 68 & 14 & 70 & 20 & 44 \\
\hline Endometriosis & 27 & 6 & 21 & 6 & 15 \\
\hline Mucus defect/dysfunction & 16 & 3 & 6 & 0 & 6 \\
\hline Sperm defect/dysfunction & 111 & 24 & 27 & 11 & 18 \\
\hline Other male infertility & 9 & 2 & 12 & 0 & 12 \\
\hline Coital/suspected coital failure & 26 & 6 & 57 & 22 & 20 \\
\hline Unexplained $\$$ & 133 & 28 & 47 & 10 & 41 \\
\hline Others & 50 & 11 & & & \\
\hline Total No of causes $\|$ & 537 & & & & \\
\hline Total $\%$ of couples & & & 41 & 11 & 29 \\
\hline
\end{tabular}

*Child=gestation at least 28 weeks, not necessarily successful.

tIncludes three women with primary ovarian failure, which is untreatable to induce ovulation.

IIncludes three women with endometriosis.

Defined by exclusion of all preceding causes, but includes some patients with minor abnormalities of doubtful importance-namely, tubal adhesions (2), endometriosis (11), and oligospermia with normal postcoital penetration of mucus (7)

athesions (2), endometriosis (11), and oligospermia with normal postcoital penetration of mucus (7). couples have two or more causes of infertility.

was $27 \%$. In the group with tubal damage ( 46 cases) the rate was $19 \%$. Those who had surgery had a conception rate of $5 \%$ and those without surgery (the less severe cases) $30 \%$.

The results for endometriosis were included in the tubal damage or unexplained groups as described above. The few cases of obstructive azoospermia were treated without success. Of the five couples with coital failure as an isolated cause, after advice four had conceived naturally within 10 months. The overall conception rate after two years in the whole clinic population with any causes of infertility was $48 \%$.

The results in the group with unexplained infertility were further analysed by the woman's age and duration of infertility when first attending the clinic (figs 2 and 3). The only significant reduction by age in the conception rate was at 35 years or more ( $51 \%$ after 18 months, being the longest period of follow up so far in this age group, compared with $66-79 \%$ in the lower age groups, $\mathrm{p}<0.01$ ). There appeared to be a progressive reduction in the chance of conception with increasing duration of infertility, although by 18 24 months of study there was no significant difference between the 1-2 year group ( 103 cases, $83 \%$ conception rate) and $2-3$ year group ( 42 cases, $84 \%$ at
18 months). After 3-5 years' infertility ( 36 cases) the conception rate after two years was reduced to $56 \%(\mathrm{p}<0.01)$, and after five years or more ( 15 cases) it was reduced still further to $30 \%(p<0.01)$. Although the data for other diagnostic groups are not given, our findings show that duration of infertility influences the subsequent chance of pregnancy only in the group with unexplained infertility.

\section{Discussion}

We have described infertility in a defined population resident in and around Bristol, which should be representative of the country as a whole and of similarly developed countries. It is not a complete picture of infertility, either in the whole population or of any fixed duration. We have described a minimum picture, expressed by the annual attendance of new couples at specialist clinics. In every case infertility had lasted at least one year; the average was $2 \frac{1}{2} 2$ years. The 
annual incidence represented $1 \cdot 2$ couples per 1000 total population. The lifetime incidence was $17 \%$-that is, about one in six couples appeared to need the help of a specialist infertility clinic at some time in their lives. One in eight needed help for a first child, and many couples would fail to have a child. Most attended gynaecology clinics, making up more than a fifth of all attendances at such clinics by residents in the administrative health district studied.

The study included primary and secondary infertility; $59 \%$ had never been pregnant, only $29 \%$ had had a child, not necessarily surviving, and $11 \%$ had had a therapeutic termination of pregnancy. Previous pregnancy appeared to contribute significantly to tubal damage causing infertility; the proportion of couples with a child was about one and a half times the average and with a previous termination twice as high. A previous termination was also twice as likely in couples with coital problems, possibly reflecting original or consequent psychological problems. Couples with unexplained

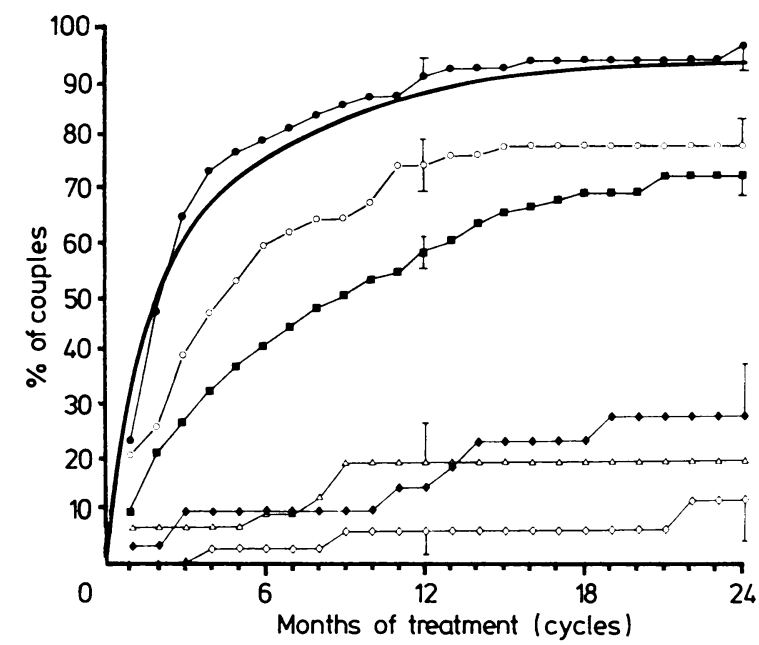

FIG l-Cumulative rates of conception in couples with single cause of infertility managed as appropriate, excluding use of donor insemination or in vitro fertilisation, compared with normal rates (highest rates reported in couples of proved fertility).${ }^{23}$ Rates for couples with each cause shown as: normal; 1 amenorrhoea; oligomenorrhoea; $\square$ unexplained infertility; $\triangle \longrightarrow \triangle$ tubal damage (moderate/severe); $\longrightarrow$ failure of sperm penetration of mucus (normal semen); $\diamond>$ oligospermia and failure to penetrate mucus. Standard error of proportions are given at 12 and 24 months.

infertility were more likely ( 1.4 times average) to have had a child, possibly reflecting the essentially chance nature of the infertility in the majority. A previous pregnancy was uncommon in women with endometriosis or mucus defects or in those with husbands having clearly defined sperm defects, suggesting a persisting inherent condition. Women who had never been pregnant were only one and a half years older on average when they started trying for a pregnancy than fertile women, suggesting that most infertility is unrelated to postponing attempts to conceive. Furthermore, age played no part in unexplained infertility at least up to 35 years (fig 2).

This study shows that the most successful treatment is for women with clearly defined ovulatory disorders, especially those with amenorrhoea (fig 1). Together with women with oligomenorrhoea they are responsible for about a fifth of infertile couples. The only unsolved problem is polycystic ovary disease. Tubal damage (excluding operative sterilisation) is a major problem, occurring in $14 \%$ of cases and with a poor chance of successful pregnancy (fig 1 ). Effectiveness of surgery is limited by irreversible endotubal and fimbrial damage. Operating on only the worst cases, as we did, seems a mistake; surgery may be better restricted to the less damaged cases. Whatever surgical policy is adopted, however, the only hope in $80 \%$ of cases would be from in vitro fertilisation.

Endometriosis was found in only $6 \%$ of cases, seldom causing tubal damage. In the absence of tubal damage the role of endo- metriosis in infertility and its treatment are not clear; recent evidence from in vitro fertilisation suggests an underlying ovarian follicular disorder. ${ }^{12}$ Primary abnormalities of secretion or function of mucus are uncommon $(3 \%)$ given normal hormonal stimulation and proper timing of collection for testing. Effective treatment has not been proved but $\mathrm{pH}$ regulation, high artificial insemination by

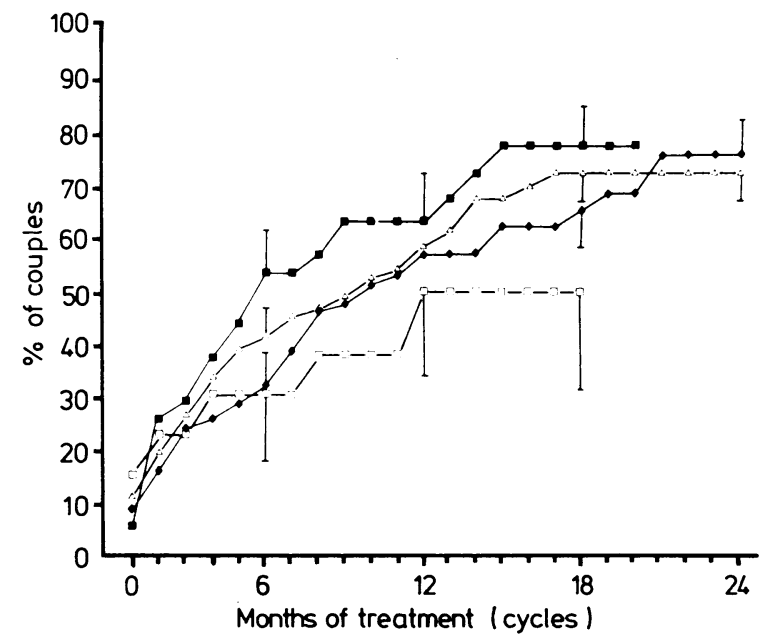

FIG 2-Cumulative rates of conception from first attendance at clinic in couples with unexplained infertility related to age of woman. Rates for each age group shown as: $\square-\square<25$ years; $\triangle \longrightarrow \triangle 25-29$ years; $30-34$ years; $\square-\square \geqslant 35$ years. Standard error of proportions are given at six, 12, 18, and 24 months.

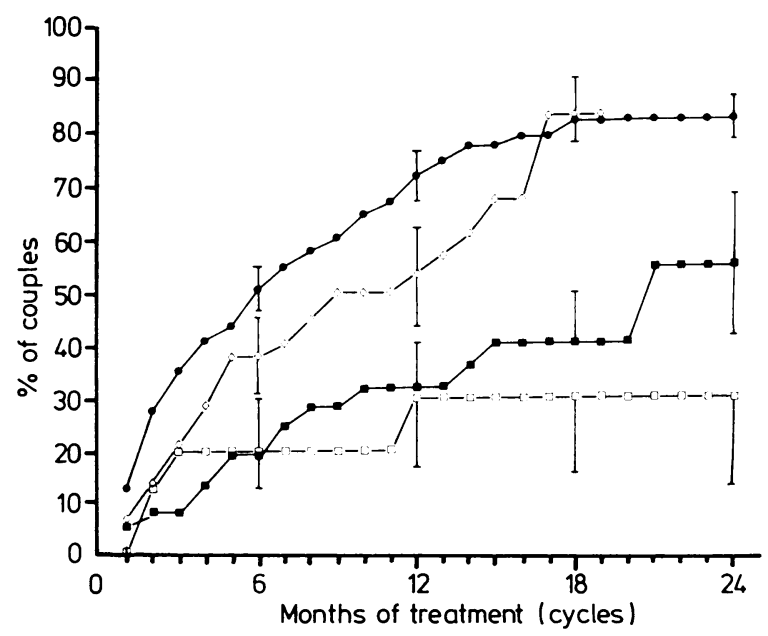

FIG 3 -Cumulative rates of conception in couples with unexplained infertility related to duration of infertility at first attendance at clinic. Rates shown as: $1-2$ years; $\diamond->2-3$ years; years; $\square-\square \geqslant 5$ years. Standard error of proportions are given at six, 12,18 , and 24 months.

the husband, and in vitro fertilisation seem possibilities. Coital failure was another uncommon cause, being evident in only $2 \%$. It was suspected in another $4 \%$ from the occurrence of normal in vitro sperm penetration of mucus despite negative postcoital testing results, and it may be relevant that the subsequent conception rate was good, although sometimes with the help of artificial insemination by the husband. ${ }^{8}$

The most commonly defined cause of infertility was defective sperm function, which occurred in a quarter of cases, and the outlook for them was poor (fig 1). The problem presented as an obvious seminal defect (oligoasthenoteratozoospermia or azoospermia) in only two thirds of this group. The remainder presented with apparently normal semen but failure of postcoital and in vitro sperm penetration of cervical mucus, the sperm defect in such cases being confirmed by study of in vitro fertilising 
ability. ${ }^{13}{ }^{14}$ On the other hand, one fifth of the men with oligospermia achieved normal sperm penetration of mucus, indicating normal sperm function, confirmed by prospective study of fertility. Inaccurate definition of male infertility is a fundamental problem common in practice and research. Penetration of mucus is an essential test, more discriminating than standard semen analysis, ${ }^{8}$ provided that valid conditions are ensured. ${ }^{7}$ Obstructive azoospermia, primary spermatogenic failure, and hypogonadotrophism seem to be uncommon additional causes of male infertility.

There is no effective treatment for most male infertility. Even after excluding patients with azoospermia the chance of natural conception amounts to only about $20 \%$ over the course of two years (fig 1), and this is not improved by artificial insemination using the husband's semen, even when inseminated high into the cervical canal and uterine cavity. ${ }^{8}$ The hopes raised by treatment with in vitro fertilisation have generally been disappointed. The only strong claim of success using in vitro fertilisation ${ }^{15}$ depended on a selectivity that remains questionable. ${ }^{16}$ With better definition of male infertility, including cases in which standard seminal analysis seems to be normal, there is likely to be increasing need for treatment by donor insemination.

Unexplained infertility gives rise to the largest group of couples, making up $28 \%$. This is not the place to discuss all the suggested causes, but clearly inclusion of laparoscopy and postcoital testing goes a long way to excluding previously unsuspected major causes. In fact the unexplained group in our study included couples with minor defects of doubtful significance, such as oligospermia (but with normal sperm penetration of mucus), minor endometriosis, or minor adhesions. The main factor determining the chance of pregnancy was the duration of infertility (fig 3 ). It seems that up to three years' failure to conceive is usually due to adversity of chance alone, and couples need only explanation and encouragement. After more than three years of unexplained infertility, and particularly after five years, the outlook is much worse (fig 3 ). In vitro fertilising capacity remains normal, however, ${ }^{12}$ and the chance of pregnancy high. ${ }^{17} \mathrm{~A}$ female factor interfering with the passage of sperms beyond the cervix seems likely, as inferred from studies using donor insemination. ${ }^{18}$

In vitro fertilisation could therefore be of real benefit to couples with prolonged unexplained infertility, who make up about a quarter of the whole unexplained group. To take these with $80 \%$ of the group with tubal damage, in vitro fertilisation would be clearly applicable in about $18 \%$ of infertile couples. This would represent about 216 new couples undergoing in vitro fertilisation each year per million of the total population. Of course, not all couples would want in vitro fertilisation, but some would want to try for a second child, and research into male infertility may extend the application of in vitro fertilisation both for treatment and diagnosis.

We are grateful to Mr J C Gingell, consultant urologist, and Dr R J M Corrall, consultant physician, for permission to study their patients. Dr Glazener was supported by a grant from the South Western Regional Health Authority Medical Research Committee.

\section{References}

1 Warnock M. Report of the committee of inquiny into human fertilisation and embryology. London HMSO, 1984

2 Tietze C. Statistical contributions to the study of human fertility. Fertil Steril 1956;7:88-95.

Tietze C. Fertility after the discontinuation of intrauterine and oral contraception. Int $\mathcal{Y}$ Fertil 1968;13:385-9.

4 Hull MGR, Savage PE, Bromham DR. Anovulatory and ovulatory infertility: results with simplified management. Br Med f 1982;284:1681-5.

5 Hull MGR, Savage PE, Bromham DR, Ismail AAA, Morris AF. The value of a single serum progesterone measurement in the midluteal phase as a criterion of a potentially fertile cycle ("ovulation") derived from treated and untreated conception cycles. Fertil Steril 1982:37: $355-60$.

6 Glazener CMA, Kelly NJ, Coulson C, et al. The prognostic value of seminal analysis in infertility. Br 7 Obstet Gynaecol 1985;92:183-4.

Hull MGR, Savage PE, Bromham DR. Prognostic value of the postcoital test: prospective study based on time-specific cumulative conception rates. Br f Obstet Grnaecol 1982;89:299-305.

8 Glazener CMA. Clinical management of the normal infertile couple. Bristol: Bristol University, 1984. (MD Thesis.)

9 Conway D, Glazener CMA, Caul EO, et al. Chlamydial serology in fertile and infertile women. Lancet 1984; i: 191-3.

10 Seibel MM, Berger MJ, W'einstein FG, Taymor ML. The effectiveness of danazol on subsequen fertility in minimal endometriosis. Fertil Steril 1982;38:534-7.

1 Hull MGR. Simplified management of anovulatory and ovulatory infertility. London: Londo University, 1983:263-5. (MD Thesis.

12 Wardle PG, Mitchell JD, McLaughlin EA, Ray BD, McDermott A, Hull MGR. Endometriosis and ovulatory disorder: reduced fertilisation in-vitro compared with tubal and unexplained infertility. Lancet 1985;ii:236-9.

13 Schats R, Aitken RJ, Templeton AA, Djahanbakhch $O$. The role of mucus-sperm interation in infertility of unknown aetiology. Br 7 Obstet (jynaecol 1984;91:371-6.

14 Hull MGR, Joyce DN, McLeod FN, Ray BD, McDermott A. Human in-vitro fertilisation, in vivo sperm penetration of cervical mucus, and unexplained infertility. Lancet 1984;ii.245-6. Cohen J Edwards RG, Fehilly $C$, et al In-vitro fertilization: a treatment for male infertility. Ferti Steril (in press

Hull MGR, Glazener CMA. Male infertility and in-vitro fertilisation. Lancet 1984;ii:231.

17 Mahadevan MM, Trounson AO, Leeton JF. The relationship of tubal blockage, infertility of unknown cause, suspected male infertility and endometriosis to success of in-vitro fertilisation
unknown unknown cause, suspected male infertility and en

18 Foss GL, Hull MGR. Results of donor insemination related to specific male infertility and unsuspected female infertility. Br $\mathcal{F}$ Obstet Gynaecol (in press).

Accepted 13.August 1985
What is the current medical view of electrolysis for removing unwanted facial hair? I have always understood that this procedure was regarded as possibly predisposing to neoplastic changes in the skin. Is this still regarded as the case?

There is absolutely no evidence whatsoever that using electrolysis to remove surplus facial hair produces neoplastic changes in the skin, and the source of this misconception is obscure. Electrolysis, whether the current is galvanic direct current or short wave alternating current, is a perfectly acceptable way of treating unwanted coarse terminal hair and is the only method which in trained hands produces permanent destruction of hair without scarring. The technique consists of introducing a fine needle into the hair follicle alongside the hair until it reaches the hair papilla, and then passing a current to destroy the growing point of the hair follicle. The current must flow for the shortest possible time to produce minimal damage, and it must flow at exactly the right place otherwise damage will be produced at the wrong site, the hair papilla will not be destroyed, the unwanted hair will still grow, and a further attempt at electrolysis will be made so that in the end more damage will be caused in the hair follicle. Too much damage produces collapse of the follicle resulting in a dimple and obvious scar at the site of the follicle.

Accordingly electrolysis to remove surplus facial hair must be undertaken by those trained and experienced in the technique. Many hairdressers, untrained and unskilled in the technique but possessing electrolysis equipment, offer this service to their clientele, who run the risk of scarring without destruction of the unwanted hair. Customers are dissatisfied, and the technique is unfairly brought into disrepute. Several reputable schools train electrologists, so those seeking treatment should ensure that their therapist is properly trained. Home electrolysis may be performed using do it yourself units bought through mail order. These units are usually galvanic and depend on the operator's own skin forming part of the electrical circuit. It is virtually impossible to insert the fine needle correctly and accurately into your own facial hair follicles even using a magnifying concave mirror, so that failure and scarring may result. Consequently, do it yourself units should never be used on the face despite manufacturers' claims for their efficacy and safety.-ALAN B SHRANK, consultant dermatologist, Shrewsbury.

A patient in her 30s developed chest pain, for which only glyceryl trinitrate gave relief. Oesophageal spasm was thought to be the cause of her pain. A few years later she developed epilepsy, spastic paralysis of the left arm, and paresis in the left leg. What might be the cause of these symptoms?

Stroke in a woman in her 30 s is extraordinarily rare, the incidence in this age group being about $0 \cdot 1 / 1000$ a year. The causes to look for are not so much related to hypertension and the complications of atheroma but include embolism from the heart, "migrinous" cerebral infarction, injury to the neck arteries, the oral contraceptive pill, or an intracranial vascular anomaly such as an arteriovenous malformation or berry aneurysm. Glyceryl trinitrate, and other vasodilators, are a fairly common cause of transient non-focal neurological symptoms (faintness, dizziness, etc) but a rare cause of focal neurological symptoms. In patients with severe occlusive arterial disease in the neck (which is usually fairly obvious and is most unlikely to be relevant in the patient here) or who have had a previous cerebral infarct, a drop in blood pressure-due to a vasodilator or any other mechanism-can cause, usually transiently, focal neurological deficits, or worsening of a previously stable neurological deficit. - C P WARLOW, clinical reader and consultant neurologist, Oxford. 